\title{
Bottom-up Synthesis of Polymeric Micro- and Nanoparticles with Regular Anisotropic Shapes
}

Ivan Lesov, ${ }^{\dagger}$ Zhulieta Valkova, ${ }^{\dagger}$ Elena Vassileva, ${ }^{\ddagger}$ George Georgiev, ${ }^{\ddagger}$ Konstans Ruseva, ${ }^{\ddagger}$ Marin Simeonov, Slavka Tcholakova, ${ }^{\dagger}$ Nikolai D. Denkov, ${ }^{\dagger}$ and Stoyan K. Smoukov ${ }^{* \dagger} \perp$ F

$\dagger$ Department of Chemical and Pharmaceutical Engineering, Sofia University, 1164 Sofia, Bulgaria

\$ Department of Pharmaceutical and Applied Organic Chemistry, Sofia University, 1164 Sofia, Bulgaria

$\perp$ Department of Materials Science \& Metallurgy, University of Cambridge, Cambridge CB30FS, UK

F School of Engineering and Materials Science, Queen Mary University of London, London E1 4NS, UK

\section{Corresponding Author}

Prof. Stoyan Smoukov

E-mail: s.smoukov@qmul.ac.uk

School of Engineering and Materials Science, Mile End Rd., Queen Mary University of London, London E1 4NS, UK 


\section{ABSTRACT}

Shape-anisotropic polymeric micro- and nanoparticles are of significant interest for the development of novel composite materials, lock-and-key assemblies, and drug carriers. Currently, syntheses require external confinement in microfluidic devices or lithographic techniques associated with significant infrastructure and low productivity, so new methods are necessary to scale-up such production efficiently. Here we report bottom-up polymerization of regular shape-anisotropic particles (polygonal platelets with different numbers of edges, with and without protruding asperities, and fibrilar particles with controllable aspect ratios), with size control over 4 orders of magnitude $(\sim 50 \mathrm{~nm}-1 \mathrm{~mm})$. Polymerization also enables the study of much smaller shapes than could previously be studied in water suspensions and we study the fundamental limits of the self-shaping transition process driving these transformations for monomer oil droplets of stearyl methacrylate (SMA) monomer oil. We show the method is compatible with a variety of polymerizing monomers and functional modifications of the particles (e.g., composites with magnetic nanoparticles, oil soluble additives, etc). We also describe post-synthetic surface modifications that lead to hierarchical superstructures. The synthesis procedure has great potential in efficient nano-manufacturing as it can achieve scalable production of the above shapes in a wide range of sizes, with minimum infrastructure and process requirements, and little maintenance of the equipment.

\section{KEYWORDS}

shape-anisotropic particles, polymeric particles, micro and nanoparticles, composite particles, magnetically functionalized particles 


\section{INTRODUCTION}

Synthesis of shape-anisotropic polymeric nano and micro-particles has been of utmost interest for the development of new functional materials, ${ }^{1-10}$ drug carriers, ${ }^{2-3}$ as well as for various applications in coating, paint production, and building industries. ${ }^{4}$ In spite of the high cost and low throughput for many current methods, such particles have demonstrated superior properties that promise creation of suspensions for inkjet printing that don't suffer from the coffee ring segregation effects, ${ }^{11}$ as well as better inhalation powder drugs (shape is important for aerodynamic properties and counterfeiting, whereas size uniformity for better dose delivery) which have currently reached stage 3 clinical trials. ${ }^{12}$ Non-spherical particles deliver not only higher surface areas, but better release of active agents contained in them, as well as better assembly and adhesion to flat or cylindrical surfaces. This makes them desirable for formulation of particle based additives into cosmetics and consumer products such as detergents and shampoos. ${ }^{13-14}$ We start by providing a brief overview of current production techniques and their relative advantages and challenges.

There are several major techniques for the synthesis of shape-anisotropic particles. ${ }^{1-31}$ Probably the most common method among them is the emulsion polymerization technique coupled with phase separation. ${ }^{15-17}$ The method is based on emulsification of a monomer or monomers mixture and their subsequent polymerization. Based on well-designed phase transitions, one could obtain various morphological modifications ranging from indented spheres to raspberrylike particles ${ }^{15}$ or even crescent-shaped particles. ${ }^{16-17}$

Alteration of crystal growth in supersaturated solutions could be used for the growth of cubic, pyramidal, spherical, ellipsoidal, rod-like, platelet-like particles. ${ }^{18-22}$ This method however, is predominantly used for inorganic particles, and can barely contribute to the shaping of polymeric 
ones. For example, the few cases available in the literature of these techniques applied to particles of organic molecules are related to the crystallization of dyes or other complex structures, rather than direct synthesis of polymers. ${ }^{23-24} \mathrm{~A}$ much more popular modification of this method is the directed precipitation of dissolved polymers via evaporation ${ }^{25-26}$ or more recently, by means of a directed flow gradient. ${ }^{27-28}$ Commonly, this modification of the method leads to the formation of polymeric fibers with different aspect ratios.

More complex shapes could be obtained via assembly of colloidal particles ${ }^{29-31}$ and physically connecting these assemblies via sintering or growth of an outer shell. ${ }^{30}$ Virtually any shape could be obtained upon elaborate planning, provided the Brownian motion of the colloids is negligible. ${ }^{31}$ These techniques need either non-scalable manual manipulation, or templates to achieve complex shapes. ${ }^{31}$

State-of-the-art techniques for control over both particles shape and dimensions, were developed by means of microfluidic devices ${ }^{1,6,7}$ and various lithography techniques. ${ }^{1,8-10}$ They all provide exceptional control, but unfortunately, include drawbacks which hinder their commercialization: (very) low productivity, demanding maintenance procedures and/or high infrastructure and production costs. ${ }^{6-10}$ There is clearly a need for the development of economically scalable and high throughput methods to make such particles that would open not only the currently envisioned but many other applications where cost has prohibited the innovative imagination.

Here, we describe a method for bottom-up synthesis of shape-anisotropic, polymeric particles with controlled shape and size. The method is based on monomer drops, which self-shape upon mild cooling ${ }^{32-34}$ and polymerize upon UV radiation. Since the process is driven by an internal 
phase transition, it requires very little infrastructure to guide the shape of the droplets. It is scalable with the volume of the emulsion used and compatible with continuous process implementations. The self-shaping allows the deformation of spherical drops into liquid polyhedra; platelets with three, four and six sides; particles with protruding asperities; and fibrils with different aspect ratios (see Figure 1). ${ }^{32,33}$ The following UV irradiation in the presence of an initiator permanently fixes the particle shape, which allows us to obtain a palette of anisotropic polymeric particles with regular shape. Here, by regular we mean that the lateral projection of the platelets presents a regular polygon (triangle, tetragon or hexagon).

The currently debated mechanism of self-shaping ${ }^{32-37}$ most probably includes the combined effect of surfactant surface freezing ${ }^{32-37}$ and subsequent templating of a sub-surface rotator phase. ${ }^{32-33,35,37-38}$ Upon cooling, a series of selected surfactants can undergo a two dimensional surface transition or surface freezing. ${ }^{32-37}$ This freezing initiates the formation of sub-surface rotator phase which in turn deforms the liquid drop. ${ }^{32-33,35,37}$ The monomer molecules in the rotator phase (aka plastic crystal) have positional order while maintaining rotational freedom around at least one axis. ${ }^{38} \mathrm{~A}$ requirement for the surface freezing and templating of this rotator phase is that the surfactant's hydrophobic tail is to have the same or longer length, compared to that of the oil, or up to 3 hydrocarbon units shorter. ${ }^{33}$

So far the rotator phase transition has been identified in: alkanes, ${ }^{32,33}$ alkenes, ${ }^{33}$ cycloalkanes, ${ }^{33}$ alcohols, ${ }^{33}$ light-responsive amines,${ }^{39}$ triglycerides,${ }^{33}$ and a wide range of multicomponent mixtures. ${ }^{35}$ Mixtures might include two or more components, that both yield rotator phases or a combination of one substance that forms a rotator phase and one that does not. The main requirement for the selection of mixtures is that the substance with higher melting temperature is more than 15 vol.\% and forms a rotator phase. ${ }^{35}$ 
The method allows scalable and efficient bottom-up production of these shapes in batch and continuous modes, with minimum equipment requirements and maintenance. ${ }^{32,33}$ Major benefits of the method include the lack of external constraints to create a shape and utilization of all the material in a droplet.

\section{EXPERIMENTAL SECTION}

Materials: All chemicals used in this study were purchased from Sigma-Aldrich. In most experiments, we used stearyl methacrylate (SMA) for preparation of emulsions and polymerization. SMA has purity of $\geq 89.5 \%$, and up to $10.5 \%$ hexadecyl methacrylate admixture. In a separate series of experiments, we used a series of alkyl acrylates with 14, 16 and 18 carbon atoms in the alkyl chain as the main phase in the polymerizing drops. Tetradecyl and hexadecyl acrylate were purchased from TCI and had $>95 \%$ and $>90 \%$ purity respectively, whereas stearyl acrylate was purchased from Sigma-Aldrich and had $>97 \%$ purity.

The nonionic surfactants Tween 40 and Tween 60 were used for stabilization of the emulsions and to assist the droplet self-shaping. According to the manufacturer, Tween 40 has a polyoxyethylene (20) sorbitan head with a mixture of C16 palmitic 90\%) and C18 stearic tails ( $10 \%)$, whereas Tween 60 has 40-60\% stearic acid tails and a total concentration of stearic and palmitic acid, $\geq 90 \%$. Both Tweens were dissolved (at e.g. $1.5 \mathrm{wt} \%$ ) in deionized water (Elix module 5, Millipore).

$\alpha$-Ketoglutaric acid, $\geq 99.0 \%$ was used for a photoinitiation of the monomer polymerization in the emulsion drops. Iron oxide, $\mathrm{Fe}_{3} \mathrm{O}_{4}$, magnetic nanoparticles solution with $20 \mathrm{~nm}$ average particle size and concentration of $5 \mathrm{mg} / \mathrm{mL}$ in toluene was purchased from Sigma-Aldrich (CAS 700304) and used for magnetic functionalization of the particles. 
Emulsion preparation: Micron-sized monodisperse emulsions were prepared using an Internal Pressure Microkit in buoyancy operated mode (SPG Technology Co., Ltd., Japan, http://www.spg-techno.co.jp/english/product/internal.shtml). We used hydrophilic cylindrical glass membranes with pore sizes of 1,3 and $10 \mu \mathrm{m}$ for preparation of drops with average diameters 3, 10 and $33 \mu \mathrm{m}$, respectively. We dissolved $1.5 \mathrm{wt} \%$ surfactant in the water phase, and started generating oil drops at the lowest transmembrane pressure (at which drop generation started) to yield monodisperse drops. Since we generated relatively large drops (which creamed) and since we did not need many drops for the capillaries, we generated emulsions with at least 1 vol.\% oil (SMA).

Submicron-emulsion droplets were prepared with high pressure homogenizer (PandaPLUS 2000, GEA). $1.5 \mathrm{~g}$ SMA was dispersed in $148.5 \mathrm{~g}$ of $1.5 \mathrm{wt} \%$ Tween 40 solution. The emulsion was passed 5 times through the high pressure homogenizer at 100 bar. The drop size distribution by volume and number is provided in SI section 6.

All emulsions were used within 2-3 weeks of their preparation and stored at temperatures around $20^{\circ} \mathrm{C}$.

Shape formation and polymerization. In a $1.5 \mathrm{~mL}$ Eppendorf tube, we mixed $0.9 \mathrm{~mL}$ of KGA (2.5 wt $\%), 0.1 \mathrm{~mL}$ surfactant solution (1.5 wt $\%)$ and $0.05 \mathrm{~mL}$ pre-made SMA emulsion (1 vol. $\%$ or more). This premix was placed in a rectangular capillary, in an isothermally adjusted chamber with precise temperature control $\left( \pm 0.2^{\circ} \mathrm{C}\right) .{ }^{[1]}$ Drop deformations were observed via optical microscopy in transmitted, cross-polarized white light (Axioplan, Zeiss, Germany). After selfshaping of the particles in the desired shapes, the initiator was activated via UV diode illumination (LTPL-C034UVH365, Liteon), set at $\approx 600 \mathrm{~mW}$. The illumination was held for 30 or 120 minutes. 
Surface modification of the particles. To enable the surface modification of the particles, we used the following procedure: In an Eppendorf tube, $1.5 \mathrm{~mL}$, we mixed $0.5 \mathrm{~mL} 5 \mathrm{wt} . \% \mathrm{KGA}, 0.5 \mathrm{~mL}$ 10 wt $\%$ Tween 40 solution, and $0.05 \mathrm{~mL}$ pre-made SMA emulsion (1 vol.\% or more). Afterwards, we polymerized the particles with UV-irradiation and left them at a constant temperature for several hours.

Composite particles formation: $0.5 \mathrm{~g}$ suspension of $\mathrm{Fe}_{3} \mathrm{O}_{4}$-in-toluene was dispersed in $2.1 \mathrm{~g}$ SMA. Afterwards, toluene was evaporated yielding $0.12 \mathrm{wt} \%$ iron oxide, dispersed in the SMA. The $\mathrm{Fe}_{3} \mathrm{O}_{4}$-in-oil suspension was emulsified via membrane emulsification or ultrasound homogenization and then used for self-shaping and polymerization. The ultrasound homogenization was made in the following way: $20 \mathrm{~mL}$ emulsion, containing 1 vol.\% SMA in $1.5 \mathrm{wt} \%$ Tween 40 was prepared via handshaking. This premix was then homogenized for $10 \mathrm{~s}$ at 400 W, using ultrasound homogenizer (SKL650-IIDN, Ningbo Haishu Sklon Development Co). Dynamic Light Scattering (DLS): The sizes of the sub-micrometer liquid drops and a reference sample of fibrilar particles was determined by using Malvern Zetasizer Nano ZS. The measurements were performed with $\lambda=633 \mathrm{~nm}$ laser in back-scattering mode, at $173^{\circ}$ scattering angle. The equipment was set to fully automated mode for measuring the hydrodynamic radius of the nanoemulsion drops, whereas the measurement time was set manually to $10 \mathrm{~s}$ per measurement for the fibrilar particles and 16 measurements were performed per point. The apparent diffusion coefficient was measured and the size of the rods was calculated as explained in SI section 6.

Electron microscopy: Scanning electron microscopy (SEM, Tescan Lyra3, Czech Republic) was used for imaging the polymeric particles larger than $300 \mathrm{~nm}$. Transmission Electron Microscopy (Jeol JEM-2100, USA) was used for measuring the particle sizes below $300 \mathrm{~nm}$ and to visualize 
the dispersed $\mathrm{Fe}_{3} \mathrm{O}_{4}$ particles in micron-sized fibrils. In both cases, the polymerized suspensions were processed for imaging immediately after their preparation. For SEM imaging we blew out the polymerized suspensions from the capillaries onto porous, cellulose filters (with nominal pore size $400 \mathrm{~nm}$ ). The surfactant and some of the smaller particles were absorbed within the filter pores, whereas larger particles remained on top of the filters. Afterwards, the particles were rinsed with a few drops of deionized water to remove the excess of surfactant. In the end, the particles were left to dry for at least 24 hours on the cellulose filters (in a clean closed box to avoid contamination with dust).

Samples for SEM were sputter-coated with 5-10 nm gold layer for imaging, whereas samples for TEM were prepared via scratching the porous filters with a spatula over a carbon-taped grid.

\section{RESULTS AND DISCUSSION}

We first demonstrate the shaping and polymerization reaction with monodisperse emulsions of stearyl methacrylate (SMA) in an aqueous solution of Tween 40 surfactant, also containing $\alpha$ ketoglutaric acid as a photoinitiator. The cooling cell was connected to a cryo-thermostate and the temperature was set to $18.9^{\circ} \mathrm{C}$. After the temperature of the cooling chamber was stabilized at $18.9^{\circ} \mathrm{C}$, the glass capillary containing the emulsion sample was carefully inserted into the cooling cell. Thus, the emulsion was subjected to a rapid cooling from room temperature down to $18.9^{\circ} \mathrm{C}$ without temperature overshooting, and it was maintained at this temperature for a long period, during which the droplets undergo shape changes (see schematics in Figure 1 and supplementary video SV1).

First, the spherical droplets deformed to polyhedra, and then to hexagonal platelets. Afterwards, they gradually changed their shape into a mixture of triangular platelets, tetragonal 
platelets, and rods or long fibers, the relative ratio of which could be controlled by the waiting time at the transformation temperature. For example, after 2 min of maintaining $18.9{ }^{\circ} \mathrm{C}$, we obtained predominantly hexagonal particles, after $15 \mathrm{~min}$ - triangular particles, and after 1 hour - fibrils. To polymerize the desired shape, we switched on a UV LED (365 nm), which triggered the reaction in the drop. Representative images of polymerized particles are shown (Figure 1, Stage 3). The shapes and yield of polymerized SMA particles could be controlled via changing the surfactant for the emulsion and the size of the initial drops (SI section 1).

The temperature of transformation, at which the emulsions are held was selected to ensure selfshaping of more than $90 \%$ of the drops in the emulsion at relatively low rate, thus allowing us to polymerize them in the desired shape along their evolution. At lower temperatures (e.g. $18^{\circ} \mathrm{C}$ ) the self-shaping process is faster and more polydisperse shapes are obtained for the platelets, but the transition to fibers is reached in just a few minutes. At even lower temperatures, e.g. $16^{\circ} \mathrm{C}$, some of the drops freeze before we can polymerize them.

To demonstrate the versatility of the method we show that we can control not only the shape but also particle size and chemical composition. Polymer shapes were obtained from three other polymerizable monomers (tetra-, hexa- and octadecyl acrylates). We found conditions where in the presence of Tween 60 surfactant and temperatures near the melting point of the oil, the droplets easily evolve in shape (e.g. SI section 2). We discuss the effects of monomer differences in the polymerization process. 
Stage 1: Emulsion preparation

drops of monomer (stearyl methacrylate)<smiles>C=C(C)C(=O)OCCCCC</smiles>

UV-initiator ( $\alpha$-ketoglutaric acid)<smiles>CCCCCCCCCC(=O)O</smiles>

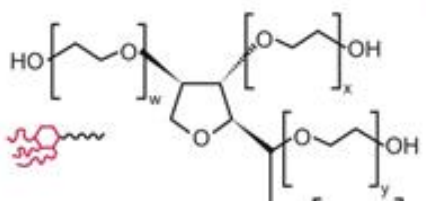

(Tween 40)
Oil droplets, dispersed in aqueous surfactant solution

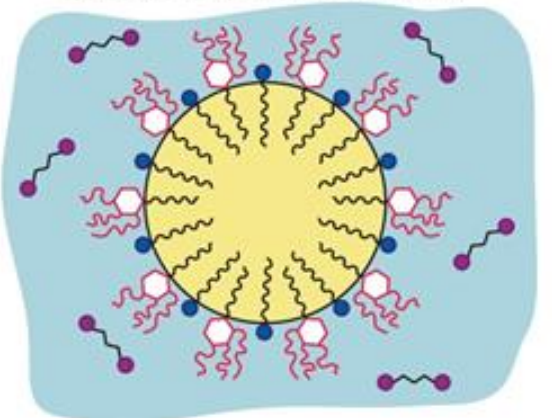

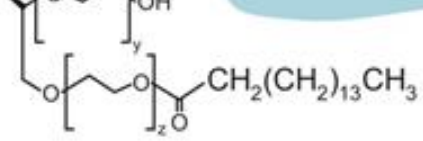

Stage 2: Monomer drops self-shaping via cooling (5 $\mu \mathrm{m}$ drops)

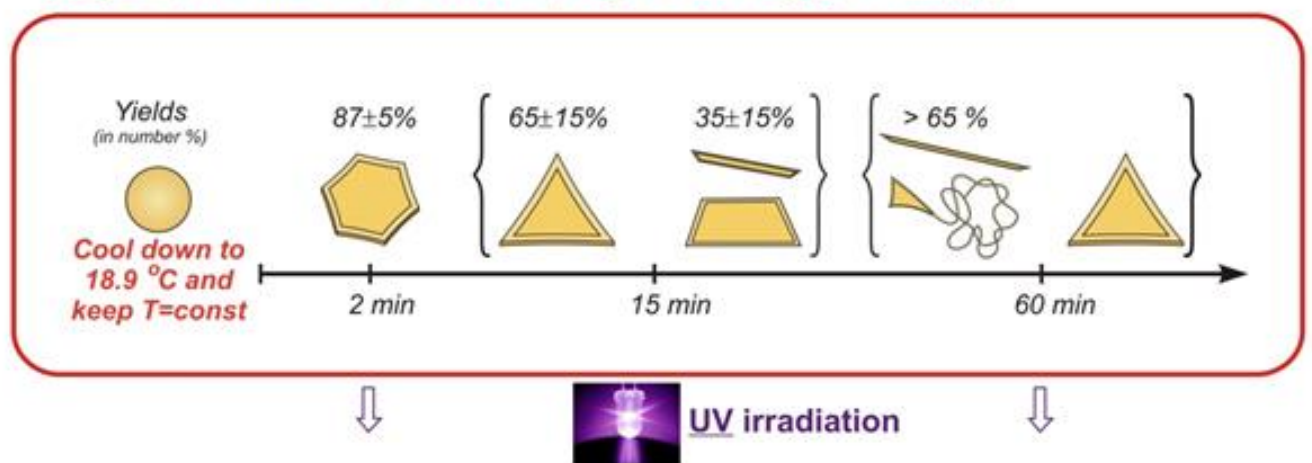

Stage 3: UV polymerization of particles of various shapes

Permanent polymer particles
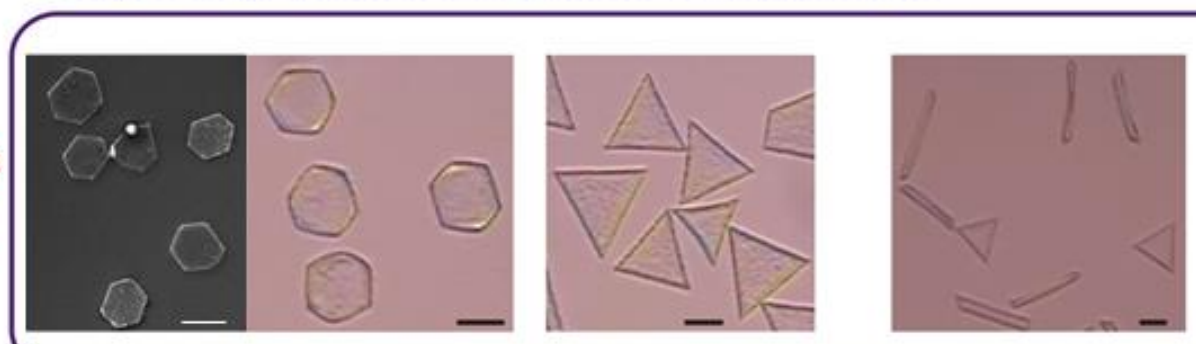

Figure 1. Stages of polymeric particles synthesis. 1) Prepare monodisperse emulsion drops, 2) cool down to reach the desired shape by self-shaping and 3) fix by UV-initiated polymerization. Horizontal bars in stage 3 are $20 \mu \mathrm{m}$. Emulsions contain 0.05 vol.\% SMA drops, $2.25 \mathrm{wt} \%$ KGA, and $0.15 \mathrm{wt} \%$ Tween 40 . 
(a)
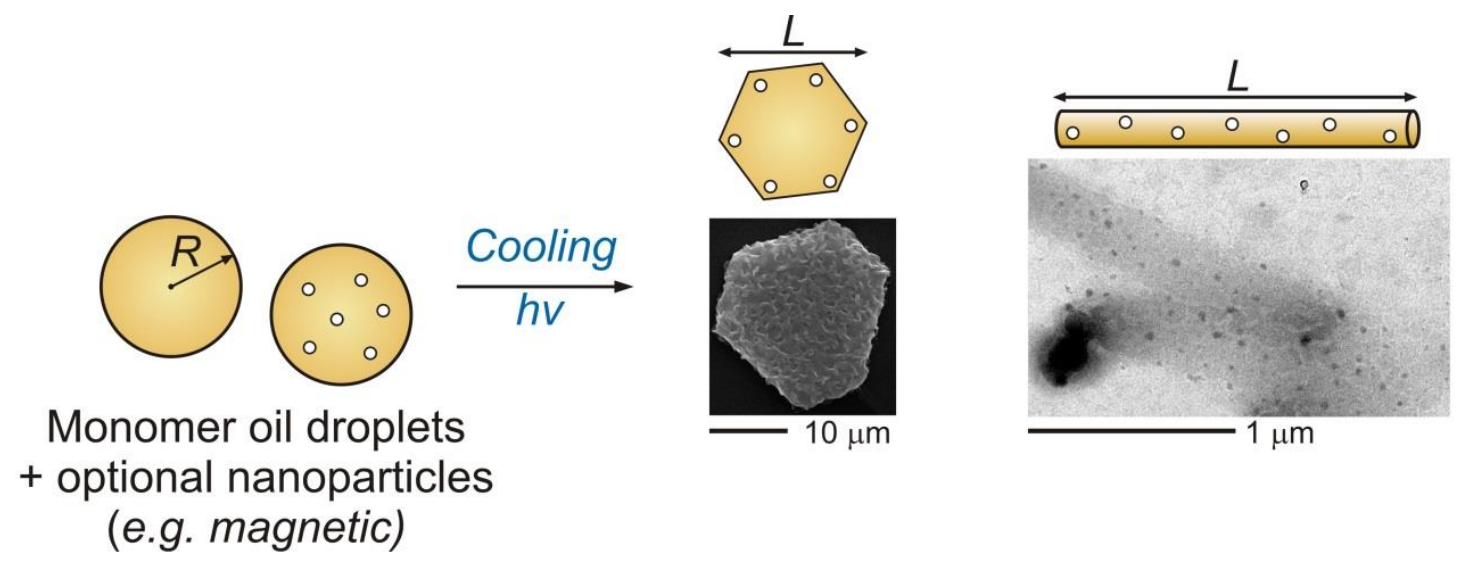

(e.g. magnetic)

(b)

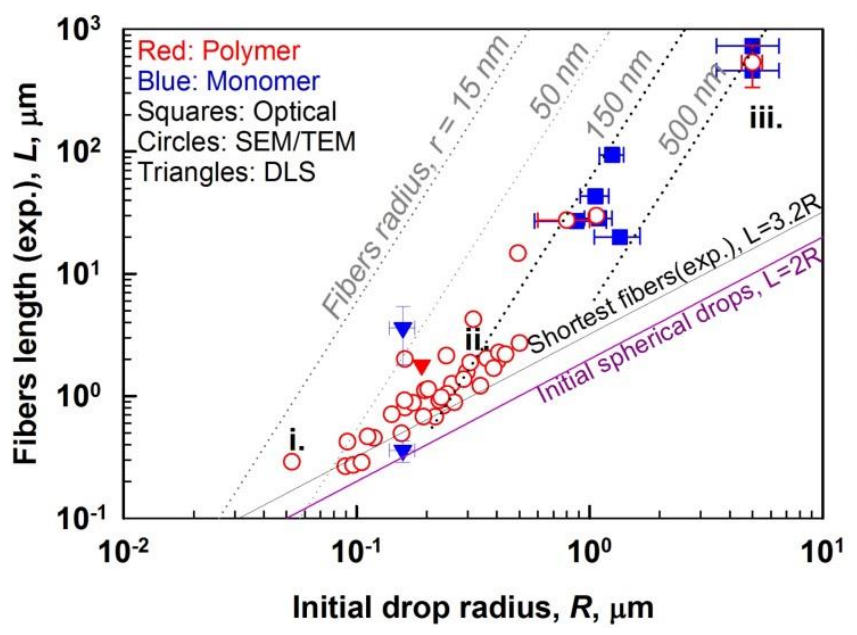

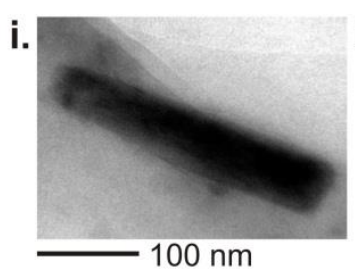
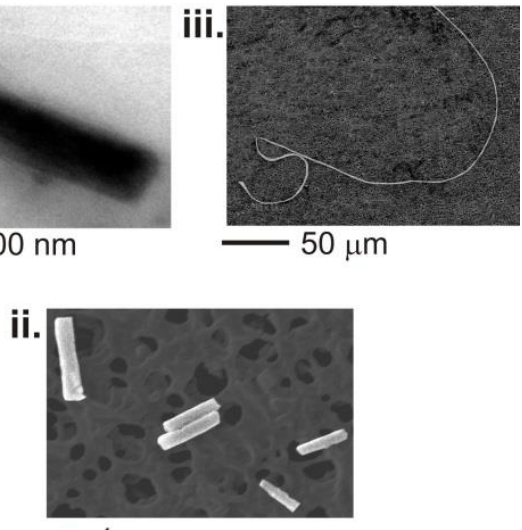

$-1 \mu \mathrm{m}$

Figure 2. (a) Preparation of poly( $\mathrm{SMA})-\mathrm{Fe}_{3} \mathrm{O}_{4}$ composite particles via self-shaping and polymerization. The figure contains SEM micrograph of a magnetic platelet, and TEM micrograph of 2 magnetic rods (the dark black spot in the image is probably a dust particle). Emulsions contain 0.05 vol.\% SMA drops, 2.25 wt\% KGA, 0.15 wt\% Tween 40 and 0.12 wt\% $\mathrm{Fe}_{3} \mathrm{O}_{4}$.(b) Experimentally determined size of SMA and poly(SMA) fibrils, as a function of the initial size of the drops. The error bars for the monomer drops represent the standard deviation of the initial droplet size, used for the preparation of the fibrils. The error bars for the polymeric fibrils are due to standard deviation in thickness determination along the whole length of the fibril. The tilted grey lines represent a geometrical estimate of the fibers thickness, assuming sphere-to-cylinder transition and volume conservation upon polymerization (i.e. negligible shrinkage). The SEM micrographs (Figure 2bii and 2biii) are digitally enhanced, as explained in SI section 3. Figure 2bi is a TEM micrograph.

In addition to changing the polymer chemistry, we found that the drop self-shaping method is compatible with the incorporation of nanoparticles in the oil phase. This greatly extends the opportunities for synthesis of multi-functional composite micro and nanoparticles with controlled 
shape (Figure 2). We show that by adding $0.12 \mathrm{wt} \% 20 \mathrm{~nm} \mathrm{Fe}{ }_{3} \mathrm{O}_{4}$ particles to SMA before emulsion preparation we can follow the same procedure for self-shaping pure SMA and obtain composite polymer particles with various shapes (Figure 2a). TEM images show the incorporation of the particles throughout the composites, and we also demonstrate the magnetization and movement of hexagonal platelets in an external magnetic field (Supplementary video SV2). Based on our recent work with multicomponent oil droplets (see ref.35), we expect the process would allow for incorporation of other oils, mixed with the polymerizable monomer. Indeed, we successfully incorporated a wide range of oily additives (as shown in the SI section 4), demonstrating great potential to tune multiple chemical functionalities for applications, such as encapsulation, drug delivery, bio and mechanical sensing, and mechanical reinforcement. The compounds tested here are mainly oil-miscible monomers that could copolymerize with SMA (methacrylic acid, vinyl acetate, 2-ethylhexyl methacrylate), crosslinking agents (trimethylolpropane methacrylate, divinylbenzene) which give rise to polymer networks, as well as oil-soluble initiator (dicumyl peroxide). Components that were initially dissolved in the oil phase, but subsequently partition between oil and water were also used for particle modification - methacrylic acid was co-polymerized with SMA, and ethylene glycol diacrylate was grafted on the surface of SA particles. The latter made the particles more rigid during heating at short time polymerizations due to crosslinking, wherein the core of the droplets remained liquid. The sizes of polymer particles fabricated by this method range from $\sim 50 \mathrm{~nm}$ to $\sim 1 \mathrm{~mm}$ scales, with various elongation ratios, $L / 2 R$ ( $L$ being the longest path along the particle, see Fig. 2A). For hexagons $L / 2 R$ is between 2 to 4 , for triangles -2.5 to 5 , and for fibers with initial radius $5 \mu \mathrm{m}$ between 3 and 80 (Figure 2b). The average thickness of the platelets could be calculated based on the elongation of the drops, when volume conservation 
is assumed (e.g. negligible shrinkage and no breakage during deformation and polymerization). Thickness was estimated to be $1.56 \pm 0.5 \mu \mathrm{m}$ for hexagonal platelets, $1.5 \pm 0.25 \mu \mathrm{m}$ for the triangular platelets and around $1.0 \mu \mathrm{m}$ for fibers, upon longer elongation times. Therefore, the average ratios between the longest length $\times$ height for drops with $R=5 \mu \mathrm{m}$ are approximately: $23 \mu \mathrm{m} \times 1.6 \mu \mathrm{m}$ for hexagons, $28 \mu \mathrm{m} \times 1.5 \mu \mathrm{m}$ for triangular platelets and between $16 \mu \mathrm{m} \times 7$ $\mu \mathrm{m}$ and $800 \times 1 \mu \mathrm{m}$ for fibers, depending on the waiting time (larger aspect ratios are obtained at longer times).

The initial droplet size was previously shown to influence the kinetics and distribution of shapes obtained from a particular oil. ${ }^{33}$ Previous optical measurements had also given some evidence near the diffraction limit that smaller droplets gave rise to smaller fiber diameters. Having polymerization allowed us to study this effect using electron microscopy.

To clarify these effects for stearyl methacrylate (SMA) and to test the ultimate limits of the self-shaping method, we prepared a series of emulsions with drop radii ranging between $50 \mathrm{~nm}$ and $5 \mu \mathrm{m}$ and polymerized them (see SI section S7). We studied quantitatively the sphere-tofiber transition for drops of initial radius $R$. Figure 2a shows their elongation (with correspondent thinning due to volume conservation) up to very long times, into cylindrical fiber structures of length, $L$ and diameter $2 r$ (aspect ratio $L / 2 r$ ). The shapes could be fixed and isolated at any stage by starting UV polymerization, and the fibril length could be controlled over more than 3 orders of magnitude $(\sim 250 \mathrm{~nm} \div 800 \mu \mathrm{m})$. Indeed, we find that smaller initial drops produced somewhat smaller in diameter fibers, but also the diameter $2 r(50 \div 1000 \mathrm{~nm})$ continues to decrease (aspect ratio increase) with time spent in cooling deformation before polymerization. 
The sharp edges of the ends of the thinnest fibrils obtained show they are likely fragments of a longer polymer fiber.

The $\approx 50 \mathrm{~nm}$ diameter of the fiber, observed in Figure 2bi, sets experimentally the lower limit for the thickness of the rotator phase. Note that this thickness is somewhat smaller than the initially anticipated one and could depend significantly on the specific system studied. ${ }^{32}$

We investigated several other effects and processes that shed light on potential ways to control and improve such bottom-up polymerization of particle shapes. The method is sensitive to molecular rearrangements during polymerization, and we observe differences between polymerizations of different monomers. For example, SMA emulsions yielded regular polymer particles, whereas acrylates yielded similar shape changes at first, but during polymerization, they exhibited various degrees of distortion. This is likely due to the coupling of volumetric shrinkage stresses developed along the polymerization and the much thinner plate geometries in the acrylate samples. Among the three acrylates, the faster rotational diffusion and reorientation ability of the smallest molecules (tetradecyl acrylate) resulted in the best preservation of the shapes in particles, as illustrated in Figure S3 in SI section 2, pointing out a potential route for optimization of regular shapes, by utilizing a series of monomers. Due to the elastic stresses, all the particles developed surface wrinkles during polymerization, ${ }^{15}$ perturbing the (initially smooth) particle surface.

Highly corrugated particles are sometimes desirable for adjusting rheological, adsorption and adhesion properties in colloids. ${ }^{40-42}$ We found we could achieve another level of structural hierarchy by controlling a post-synthetic separate process for modifying the surface, which as to our knowledge has not been observed until now, see Figure 3. 
(a)

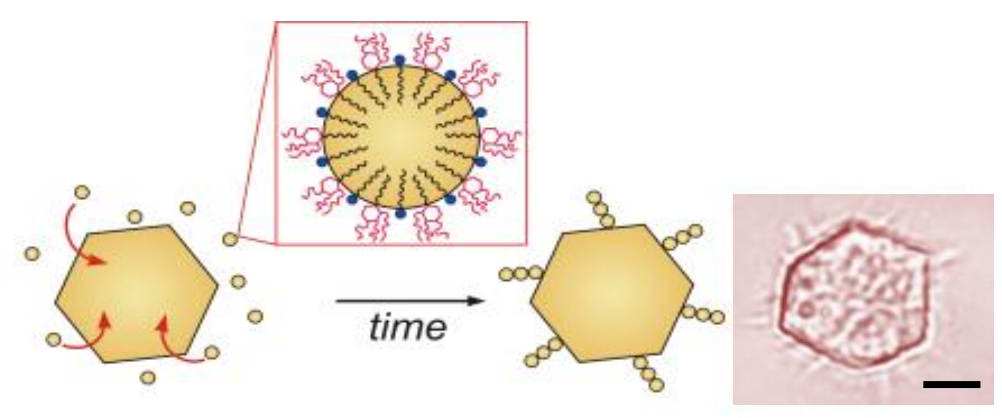

(b)
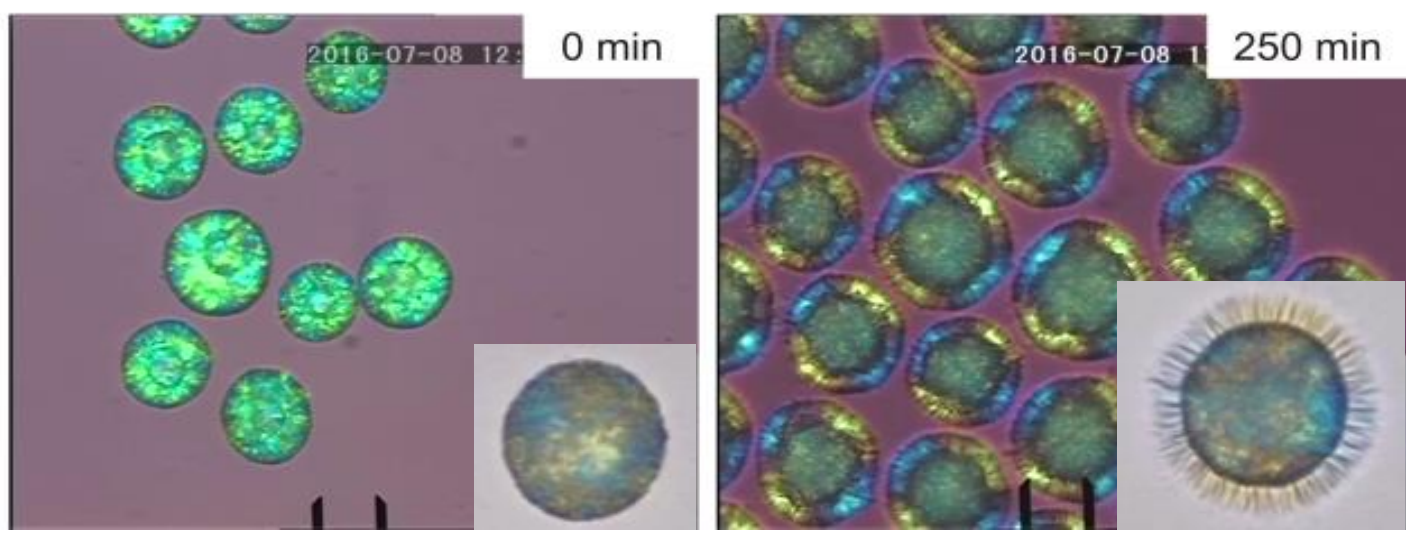

Figure 3. (a) Morphological modification of particles surface: (a) Case of single anisotropic particle and hypothetical mechanism. Scale bars are $5 \mu \mathrm{m}$.(b) Kinetics of spikes growth in presence of higher surfactant concentration and spherical particles, $5 \mathrm{wt} \%$ Tween 40, $2.5 \mathrm{wt} \%$ ketoglutaric acid and $0.05 \mathrm{wt} \%$ SMA. The scale bars are $20 \mu \mathrm{m}$.

A "crown" of spikes was observed to gradually grow on the particle surface when particles were left to polymerize for a long time (Figure 3a). The spike length and surface density increased with time until the whole surface of the particles was covered by a dense layer of micrometer long asperities. We hypothesized this behavior may be related to surfactantmonomer aggregates (mixed micelles) formed in the aqueous phase. The monomers polymerize in the interior of the micelles, forming nanoclusters of poly-SMA, and later attach to the already polymerized micrometer particles. We tested this hypothesis by increasing the concentration of Tween 40 surfactant in the aqueous phase to 5 and $10 \mathrm{wt} \%$, to increase the amount of monomers solubilized in micelles. Afterwards, we polymerized the emulsion drops at $33^{\circ} \mathrm{C}$. As a result of 
the small size of the poly-SMA clusters formed in the micelles (e.g. in the range between 3 and $12 \mathrm{~nm}$, see SI section 5), these clusters started self-assembling into large spikes upon supercooling to $18.9^{\circ} \mathrm{C}$. The size of the spikes increased with the increase of surfactant concentration, and their growth was faster at higher surfactant concentrations (see Figure $3 \mathrm{~b}$ and SI section 5). The latter trends support our hypothesis that the spikes are formed by selfassembly of nano-clusters of poly-SMA, formed inside the surfactant micelles.

\section{CONCLUSIONS}

We have introduced a novel bottom-up method for synthesizing nanometer to millimeter size particles of a variety of regular anisotropic shapes, sizes, and chemical compositions. Drops of reactive monomers were shaped into desired structures and polymerized to fix the shape permanently by UV irradiation. We outline the factors for controlling the yield of different shapes (emulsion surfactant, initial droplet size, and time in cooling stage). Regularly shaped hexagonal and triangular platelets, and fibrilar particles were produced from droplets as small as $50 \mathrm{~nm}$, and with control over size from $50 \mathrm{~nm}$ to $1 \mathrm{~mm}$ in at least one of their dimensions.

Functional composite particles were obtained by incorporation of (e.g. magnetic) nanoparticles in the monomer oil droplets, and by co-polymerizing mixtures of reactive oil monomers (e.g. with cross-linking agents), allowing chemical customization of the shaped particles. Postsynthetic modification of the particles surface could also be attained via the reported selfassembly of nano-crystallites or by the classical methods of polymer and/or surfactant adsorption. Such modifications could be used to tune the particle interactions with other 
particle/surfaces, opening the prospect for self-assembly of larger, macroscopic hierarchical structures.

\section{ACKNOWLEDGMENTS}

The authors gratefully acknowledge the financial support of grants to Stoyan Smoukov EMATTER (\# 280078) and Proof-of-Concept ShipShape (\# 766656) by the European Research Council (ERC), and by the Scientific Research Fund of Sofia University (Project No. 80-10225). The study falls under the umbrellas of European network COST MP 1305 and the Horizon 2020 project “Materials Networking” (ID: 692146-H2020-eu.4.b).

\section{ASSOCIATED CONTENT}

Supporting Information

Additional experimental results for the preparation of anisotropic particles with different surfactants and different drops sizes, examples of polymerization of particles with different compositions, as well as modification of particle surface morphology are provided in the Supplementary Information.

Supplementary video 1 - self-shaping of particles presented in Figure 1; experimental conditions: $5 \mu \mathrm{m}$ SMA drops in $2.25 \mathrm{wt} \% \mathrm{KGA}$, and $0.15 \mathrm{wt} \%$ Tween 40 . Scale bars are $50 \mu \mathrm{m}$ Supplementary video 2 - hexagonal platelets movement in magnetic field Supplementary video 3 -reversible modification of particle surface morphology upon heating The Supporting Information is available free of charge on the ACS Publications website at DOI: 


\section{AUTHOR INFORMATION}

\section{Corresponding Author}

Prof. Stoyan Smoukov

E-mail: s.smoukov@qmul.ac.uk

School of Engineering and Materials Science, Mile End Rd., Queen Mary University of London, London E1 4NS, UK 


\section{REFERENCES}

(1) Dendukuri, D; Doyle, P.S. The Synthesis and Assembly of Polymeric Microparticles Using Microfluidics, Adv. Mater. 2009, 21, 407.

(2) Champion, J.A.; Katare, Y.K.; Mitragotri, S. Particle shape: a new design parameter for micro- and nanoscale drug delivery carriers. J. Control. Release 2007, 121, 3.

(3) Perry, J.L.; Herlihy, K.P.; Napier, M.E., DeSimone, PRINT: A Novel Platform Toward Shape and Size Specific Nanoparticle Theranostics, J.M. Acc. Chem. Res. 2011, 44, 990.

(4) Gokmen, M.T.; Du Prez, F.E. Porous polymer particles: a comprehensive guide to synthesis, characterization, functionalization and applications, Prog. Polym. Sci. 2012, 37, 365.

(5) Champion, J. A.; Katare, Y.K.; Mitragotri, S. Making polymeric micro- and nanoparticles of complex shapes, Proc. Natl. Acad. Sci. 2007, 104, 11901.

(6) Xu, S.; Nie, Z.; Seo, M.; Lewis, P.; Kumacheva, E.; Stone, H.A.; Garstecki, P.; Weibel, D.B.; Gitlin, I.; Whitesides, G.M. Generation of Monodisperse Particles by Using Microfluidics: Control over Size, Shape, and Composition, Angew. Chemie Int. Ed. 2005, 44, 724.

(7) Shum, H.C.; Abate, A.R.; Lee, D.; Studart, A.R.; Wang, B.; Chen, C.-H.; Thiele, J.; Shah, R.K.; Krummel, A.; Weitz, D.A. Droplet microfluidics for fabrication of non-spherical particles, Macromol. Rapid Commun. 2009, 31, 108.

(8) Dendukuri, D.; Pregibon, D.C.; Collins, J.; Hatton, T.A.; Doyle, P.S. Continuous-flow lithography for high-throughput microparticle synthesis. Nat. Mater. 2006, 5, 365. 
(9) Gratton, S.E.; Ropp, P.; Pohlhaus, P.D.; Luft, J.C.; Madden, V.J.; Napier, M.E.;

DeSimone, The effect of particle design on cellular internalization pathways, J.M. Proc. Natl. Acad. Sci. 2008, 105, 11613.

(10) Choi, C.-H.; Lee, J.; Yoon, K.; Tripathi, A.; Stone, H.A.; Weitz, D.A.; Lee, C.-S. Surface-Tension-Induced Synthesis of Complex Particles Using Confined Polymeric Fluids, Angew. Chemie Int. Ed. 2010, 49, 7748.

(11) Yunker PJ , Still T, Lohr MA \& Yodh AG, Suppression of the coffee-ring effect by shapedependent capillary interactions, Nature 2011, 476, p 308.

(12) https://clinicaltrials.gov/ct2/show/NCT03399604, Accessed on 29 May, 2018

(13) Bayles, A. V; Prileszky, T. A.; Spicer, P. T.; Furst, E. M. Model of Structured Emulsion Droplet Stability and Reconfigurability. Langmuir 2018, 34 (13), 4116-4121.

(14) Spicer, P.T., et al. Non-spherical droplet, US Patent 9,597.648 B2 (2017)

(15) Peng, B.; Imhof, A. Surface Morphology Control of Cross-Linked Polymer Particles via Dispersion Polymerization. Soft Matter 2015, 11 (18), 3589-3598.

(16) Fan, J.-B.; Liu, H.; Song, Y.; Luo, Z.; Lu, Z.; Wang, S. Janus Particles Synthesis by Emulsion Interfacial Polymerization: Polystyrene as Seed or Beyond? Macromolecules 2018, 51 (5), 1591-1597.

(17) Fan, J.-B.; Song, Y.; Liu, H.; Lu, Z.; Zhang, F.; Liu, H.; Meng, J.; Gu, L.; Wang, S.; Jiang, L. A General Strategy to Synthesize Chemically and Topologically Anisotropic Janus Particles. Sci. Adv. 2017, 3 (6). 
(18) Rossi, L.; Sacanna, S.; Irvine, W. T. M.; Chaikin, P. M.; Pine, D. J.; Philipse, A. P. Cubic Crystals from Cubic Colloids. Soft Matter 2011, 7 (9), 4139-4142.

(19) Wang, P.; Yang, Y.; Zhuang, J.; Wang, X. Self-Adjustable Crystalline Inorganic Nanocoils. J. Am. Chem. Soc. 2013, 135 (18), 6834-6837.

(20) Xiao, J.; Qi, L. Surfactant-Assisted, Shape-Controlled Synthesis of Gold Nanocrystals. Nanoscale 2011, 3 (4), 1383.

(21) Peng, X.; Manna, L.; Yang, W.; Wickham, J.; Scher, E.; Kadavanich, A.; Alivisatos, A. P. Shape Control of CdSe Nanocrystals. Nature 2000, 404, 59.

(22) Shchepelina, O.; Kozlovskaya, V.; Singamaneni, S.; Kharlampieva, E.; Tsukruk, V. V. Replication of Anisotropic Dispersed Particulates and Complex Continuous Templates. J. Mater. Chem. 2010, 20 (32), 6587.

(23) Taden, A.; Landfester, K.; Antonietti, M. Crystallization of Dyes by Directed Aggregation of Colloidal Intermediates: A Model Case. Langmuir 2004, 20 (3), 957-961.

(24) Huang, M.; Antonietti, M.; Cölfen, H. Morphology-Controlled Growth of Perylene Derivative Induced by Double-Hydrophilic Block Copolymers. APL Mater. 2016, 4 (1), 015705.

(25) M. Su, Z. Su, Effects of Solvent Evaporation Rate and Poly(acrylic acid) on Formation of Poly(ethylene oxide)- block -polystyrene Micelles from Emulsion, Macromolecules. 47 (2014) 1428-1432. 
(26) Nie, X.; Zhang, Y.; Wang, M.; Jiang, W. A Versatile Approach to Prepare Ultralong Nanofibers by Coassembly of Block Copolymers and Nanoparticles in Emulsions. New J. Chem. 2016, 40 (5), 4556-4561.

(27) Smoukov, S. K.; Tian, T.; Vitchuli, N.; Gangwal, S.; Geisen, P.; Wright, M.; Shim, E.; Marquez, M.; Fowler, J.; Velev, O. D. Scalable Liquid Shear-Driven Fabrication of Polymer Nanofibers. Adv. Mater. 2015, 27 (16), 2642-2647.

(28) Zander, E. N.; Gillan, M.; Sweetser, D. Composite Fibers from Recycled Plastics Using Melt Centrifugal Spinning. Materials (Basel). 2017, 10 (9), 1044.

(29) Sacanna, S.; Pine, D. J. Shape-Anisotropic Colloids: Building Blocks for Complex Assemblies. Curr. Opin. Colloid Interface Sci. 2011, 16 (2), 96-105.

(30) Ni, S.; Marini, E.; Buttinoni, I.; Wolf, H.; Isa, L. Hybrid Colloidal Microswimmers through Sequential Capillary Assembly. Soft Matter 2017, 13 (23), 4252-4259.

(31) Ni, S.; Isa, L.; Wolf, H. Capillary Assembly as a Tool for the Heterogeneous Integration of Micro- and Nanoscale Objects. Soft Matter 2018, 14 (16), 2978-2995.

(32) Denkov, N.; Tcholakova, S.; Lesov, I.; Cholakova, D.; Smoukov, S. K. Self-Shaping of Oil Droplets via the Formation of Intermediate Rotator Phases upon Cooling. Nature 2015, 528 (7582), 392-395.

(33) Cholakova, D.; Denkov, N.; Tcholakova, S.; Lesov, I.; Smoukov, S. K. Control of Drop Shape Transformations in Cooled Emulsions. Adv. Colloid Interface Sci. 2016, 235. 
(34) Guttman, S.; Sapir, Z.; Schultz, M.; Butenko, A. V.; Ocko, B. M.; Deutsch, M.; Sloutskin, E. How Faceted Liquid Droplets Grow Tails. Proc. Natl. Acad. Sci. 2016, 113 (3), 493-496.

(35) Cholakova, D.; Valkova, Z.; Tcholakova, S.; Denkov, N.; Smoukov, S. K. "Self-Shaping" of Multicomponent Drops. Langmuir 2017, 33 (23), 5696-5706.

(36) Guttman, S.; Sapir, Z.; Ocko, B. M.; Deutsch, M.; Sloutskin, E. Temperature-Tuned Faceting and Shape Changes in Liquid Alkane Droplets. Langmuir 2017, 33 (5), 1305-1314.

(37) Denkov, N.; Cholakova, D.; Tcholakova, S.; Smoukov, S. K. On the Mechanism of Drop Self-Shaping in Cooled Emulsions. Langmuir 2016, 32 (31), 7985-7991.

(38) Shinohara, Y.; Takamizawa, T.; Ueno, S.; Sato, K.; Kobayashi, I.; Nakajima, M.; Amemiya, Y. Microbeam X-Ray Diffraction Analysis of Interfacial Heterogeneous Nucleation of n Hexadecane inside Oil-in-Water Emulsion Droplets. Cryst. Growth Des. 2008, 8 (9), 3123-3126.

(39) Martin, N.; Sharma, K. P.; Harniman, R. L.; Richardson, R. M.; Hutchings, R. J.; Alibhai, D.; Li, M.; Mann, S. Light-Induced Dynamic Shaping and Self-Division of Multipodal Polyelectrolyte-Surfactant Microarchitectures via Azobenzene Photomechanics. Sci. Rep. 2017, $7,41327$.

(40) Zanini, M.; Marschelke, C.; Anachkov, S. E.; Marini, E.; Synytska, A.; Isa, L. Universal Emulsion Stabilization from the Arrested Adsorption of Rough Particles at Liquid-Liquid Interfaces. Nat. Commun. 2017, 8, 15701. 
(41) Fernandez, N.; Mani, R.; Rinaldi, D.; Kadau, D.; Mosquet, M.; Lombois-Burger, H.; Cayer-Barrioz, J.; Herrmann, H.J.; Spencer, N.D.; Isa, L. Microscopic Mechanism for Shear Thickening of Non-Brownian Suspensions, Phys. Rev. Letters 2013, 111, 108301

(42) Xu, D.; Wang, M.; Ge, X. X.; Hon-Wah Lam, M.; Ge, X. X. Fabrication of Raspberry SiO2/Polystyrene Particles and Superhydrophobic Particulate Film with High Adhesive Force. J. Mater. Chem. 2012, 22 (12), 5784. 


\section{For Table of Contents use only}

Title: Bottom-up Synthesis of Polymeric Micro- and Nanoparticles with Regular Anisotropic Shapes

Authors: Ivan Lesov, Zhulieta Valkova, Elena Vassileva, George Georgiev, Konstans Ruseva, Marin Simeonov, Slavka Tcholakova, Nikolai D. Denkov, and Stoyan K. Smoukov

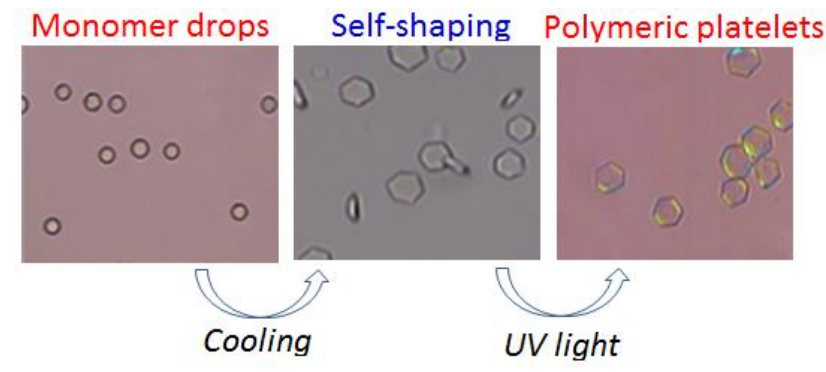

